\section{Marks Fewest Reported Measles Cases in U.S.}

As of July 3, 1993, (week 26), local and state health departments reported a provisional total of 167 measles cases for 1993-the lowest total reported for the first 26 weeks of any year since surveillance began in 1943. The represents a $99 \%$ decrease from the 13,787 cases reported during the first 26 weeks of 1990, the peak of the recent resurgence.

Overall, 39 reported case patients $(22 \%)$ had received one dose of measles-containing vaccine on or after their first birthday; no cases were reported among persons who had received two doses of vaccine. A total of 47 reported case patients (27\%) were unvaccinated but vaccine eligible (ie, U.S. citizens aged $\geqslant 16$ months without medical, religious, or philosophical exemption to vaccination). Other unvaccinated groups included 3 persons $(20 \%)$ with philosophical exemptions, 30 (17\%) who were aged $<16$ months, $10(6 \%)$ who were born before 1957, and $10(6 \%)$ who were non-U.S. citizens.

During 1993, measles cases have involved predominantly school-aged persons, and the largest outbreaks have occurred among school-aged children who received one dose of measles vaccine (ie, vaccine failures). In contrast, from 1989 through 1991, cases involved predominantly preschool-aged children, and the largest outbreaks occured among unvaccinated preschool-aged children living in large urban areas.

The decline in measles incidence during 1992 and 1993 most likely reflects increased measles vaccination coverage levels among preschool-aged children. The estimated level of measles vaccination coverage for children aged two years was substantially higher in $1991(83 \%)$ than in $1985(61 \%)$. The decline also may reflect a decrease in measles importation from other countries in the Western hemisphere associated with aggressive measles control programs.

Although the low reported incidence of measles in 1993 suggests transmission has been interrupted in many parts of the United States, the report of 102 indigenous cases without a known source indicates that undetected transmission still is occuring in some areas.

FROM: Centers for Disease Control and Prevention. M M W R 1993:42:813-816

\section{Nationwide Study of Latex Allergies in HCWs}

The Health Industry Manufacturers Association (HIMA) and the Centers for Disease Control and Prevention (CDC) are collaborating on a study of reactions to latex-containing products to determine the prevalence and type of natural rubber latex reactions among workers at healthcare facilities and latex manufacturing plants nationwide.

Reports of adverse reactions to products containing latex have been increasing both in the medical literature and in the Medical Device Accident Report System at the Food and Drug Administration (FDA). These reports have identified healthcare workers and pediatric patients with myelodysplasia and other congenital abnormalities as high-risk groups for adverse reactions to products containing natural rubber latex. However, no such reports have occurred among natural rubber latex manufacturing workers.

Only two nationwide surveys of healthcare workers have been conducted to determine the prevalence of reactions to latex-containing products. One study by the Association of Operating Room Nurses (AORN) (in press), in collaboration with the CDC, surveyed operating room nurses, and another study surveyed army dentists (JAMA 1992;268:2695). However, the prevalence of reactions to products containing natural rubber latex among all types of healthcare workers and among latex manufacturing workers is unknown.

HIMA and CDC are soliciting healthcare facilities and organizations to participate in this study. For more information on this project, contact Siiri N. Bennett, MD, CDC, Hospital Infections Program, Mailstop A-07, 1600 Clifton Rd. NE, Atlanta GA 30333. Telephone (404) 639-1550 or FAX (404) 639-3770.

\section{Valaciclovir More Effective Than Acyclovir in Reducing Pain from Shingles}

Patients with shingles who were treated with the experimental drug valaciclovir were found to have one to two weeks less herpetic pain than patients treated with acyclovir, according to Dr. Karl Beutner at the University of California, San Francisco. The results from an international, double-blind randomized study involving more than 1,100 adults was presented at the 33rd Interscience Conference on Antimicrobial Agents and Chemotherapy (ICAAC) held on October 17-20, 1993, in New Orleans.

Shingles, also called herpes zoster, is caused by the reactivation of the varicella zoster virus acquired from an earlier episode of chickenpox.

Valaciclovir is an ester of acyclovir and is converted rapidly to acyclovir after oral administration. Because it is absorbed significantly better than acyclovir when taken orally, it requires fewer daily doses and may be more effective. Valaciclovir is being studied to define its safety and efficacy for individuals 